\title{
Linear Quadratic Servo Design for Magnetic Levitation Systems Considering Disturbance Forces from Linear Synchronous Motor
}

\author{
Chang-Hyun Kim*, Hanwoong Ahn**, Ju Lee*** and Hyungwoo Lee ${ }^{\dagger}$
}

\begin{abstract}
Recently, the demand of maglev systems in the manufacturing industry for LCD and OLED display panels, which are required to be very clean and possess vacuum systems, has been increasing due to their characteristics such as being non-contact, noise free and eco-friendly. However, it is still a challenge to simultaneously control both the propulsion and levitation for their interactive effect difficult to be exactly measured. In this paper, we proposed a new tuning method for controlling the magnetic levitation force robustly against the levitation disturbance caused by a propulsion system, based on LQ servo optimal control. The disturbance torque of the LSM propulsion system is calculated through FEM analysis in such a way that the LQ servo controller is determined in order to minimize the effect of the disturbance. The robust performance of the proposed LQ servo control method for the in-track type magnetic levitation systems is demonstrated via simulations and experiments.
\end{abstract}

Keywords: Maglev, Multi-objective, LQ servo, Electromagnetic suspension

\section{Introduction}

Since maglev systems are contactless systems, they are suitable for high-speed transport systems. In addition, it produces significantly less noise pollution due, its maintenance cost is low and it is currently one of the more popular industries because it is eco-friendly [1].

Magnetic levitation systems can be classified as follows: EMS(Electro Magnetic Suspension), EDS (Electro Dynamic Suspension), superconducting electrodynamic using a superconductor, and Hybrid-EMS with a permanent magnet inserted into the levitation electromagnet, depending on the levitation force[2]. The Hybrid-EMS method, which uses a permanent magnet, is not suitable because the supply and demand rate of a clean distribution transport system is sensitively determined by the electronic device near to the product. Furthermore, the repulsive levitation device is characterized by a stable system, but precise levitation control is difficult; thus attractive levitation methods can produce a smaller levitation resolution compared to repulsive levitation methods. In this paper, we focus on the levitation control with respect to EMS of Maglev system.

For the levitation control of the in-track type Maglev systems, one of the most difficult problems is how to design the controller reducing the effect of propulsion systems. Since the levitation disturbance force from the

$\dagger$ Corresponding Author: Dept. of Railway Vehicle System Engineering, Korea National University of Transportation, Korea. (krhwlee@ut.ac.kr)

* Dept. of Electricity, VISION College of Jeonju, Korea. (chkim@jvision.ac.kr)

** Satellite Control System Team, Korea Aerospace Research Institute, Korea. (ahnhw@kari.re.kr)

*** Dept. of Electrical Engineering, Hanyang University, Korea. (julee@hanyang.ac.kr)

Received: April 4, 2016; Accepted: October 28, 2016
LSM is hard to be measured, it is exceedingly difficult to design a controller for the EMS maglev system [3]. This paper deals with the LSM levitation disturbance through finite element method FEM analysis.

The EMS maglev system has a structure for controlling and maintaining constant levitation by sensing or estimating parameters, such as the distance of levitation, speed, and electric current. Generally, a proportion integration difference PID control is often used for levitation control. Since the PID control does not require a state estimation of the levitation system, it is simple to realize. However, it is vulnerable to noise and there is a limit to enhancing the fast-response [4]. In this paper, we study the design of an LQ servo control system that is resistant against such disturbances. In addition, a disturbance resulting from LSM analysis was analyzed using FEM analysis. Furthermore, we modelled the maglev system and a performance comparison of the PID control and LQ servo control has been conducted through simulation and examination.

\section{Model of Maglev}

The EMS maglev system has produced levitation from

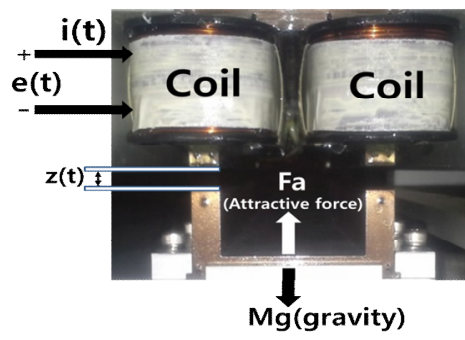

Fig. 1. Construction of electromagnetic suspension systems 
an attractive force between the rail tracks and magnets. The EMS system with general U-shape electromagnetics is depicted in Fig. 1 [5]. In the Fig. 1, upper side of the core is the fixed rail track and lower $U$ shape iron core is the moving vehicle, called as in-track type (passive tray) MagLev systems.

The voltage equation of the EMS-type electromagnetic suspension system can be expressed as [6]

$$
\frac{d i(t)}{d t}=-\frac{2 z(t)}{\mu_{0} N^{2} A} R i(t)+\frac{i(t)}{z(t)} \frac{d z(t)}{d t}+\frac{2 z(t)}{\mu_{0} N^{2} A} e_{a}(t)
$$

where $i(t)$ is the current in the coil, $z(t)$ is the gap position, $e_{a}(t)$ is the applied voltage, $\mu_{0}$ is the permeability of air gap, $N$ is the number of coil turns, $A$ is the pole area of the coil, and $R$ is the resistance of the coil.

According to Newton's second law of motion, the motion equation of EMS-type electromagnetic suspension system is as follows:

$$
\frac{d^{2} z(t)}{d t^{2}}=g-\frac{\mu_{0} N^{2} A}{4 M}\left(\frac{i(t)}{z(t)}\right)^{2}+\frac{1}{M} F_{d}(t)
$$

where $g$ is the gravitational constant, $M$ is the mass of the levitated vehicle, and $F_{d}(t)$ is the external force (disturbance) generated by external factors. In this paper, we consider $F_{d}(t)$ as levitation disturbance force caused by the propulsion system. The nonlinear mathematical model of the Maglev system can be linearized at the operating point $\left(z_{o}, \dot{z}_{o}, i_{o}, e_{o}, F_{d o}\right)$ as follows $[7,8]$

$$
\begin{aligned}
{\left[\begin{array}{l}
\Delta \dot{x}_{1}(t) \\
\Delta \dot{x}_{2}(t) \\
\Delta \dot{x}_{3}(t)
\end{array}\right] } & {\left[\begin{array}{ccc}
0 & 1 & 0 \\
\frac{\mu_{0} N^{2} A}{2 M} \frac{i_{0}^{2}}{z_{0}^{3}} & 0 & -\frac{\mu_{0} N^{2} A}{2 M} \frac{i_{0}}{z_{0}^{2}} \\
-\frac{2 R i_{0}}{\mu_{0} N^{2} A}-\frac{i_{0} \dot{z}_{0}}{z_{0}^{2}}+\frac{2 e_{a 0}}{\mu_{0} N^{2} A} & \frac{i_{0}}{z_{0}}-\frac{2 R i_{0}}{\mu_{0} N^{2} A}+\frac{\dot{z}_{0}}{z_{0}^{2}}
\end{array}\right]\left[\begin{array}{l}
\Delta x_{1}(t) \\
\Delta x_{2}(t) \\
\Delta x_{3}(t)
\end{array}\right] } \\
& +\left[\begin{array}{c}
0 \\
0 \\
\frac{2 z_{0}}{\mu_{0} N^{2} A}
\end{array}\right] \Delta u(t)+\left[\begin{array}{c}
1 \\
M \\
0
\end{array}\right] \Delta F_{d}(t)
\end{aligned}
$$

where

$$
\begin{aligned}
& \Delta x_{1}(t)=z(t)-z_{0}, \Delta x_{2}(t)=\dot{z}(t)-\dot{z}_{0}, \Delta x_{3}(t)=i(t)-i_{0}, \\
& \Delta u(t)=e_{a}(t)-e_{a 0} \text { and } \Delta F_{d}(t)=F_{d}(t)-F_{d 0} .
\end{aligned}
$$

If it is assumed that the electric current is the input of the EMS which is actuated sufficiently faster than the motion equation, then the voltage equation can be omitted as follows $[3,6]$ :

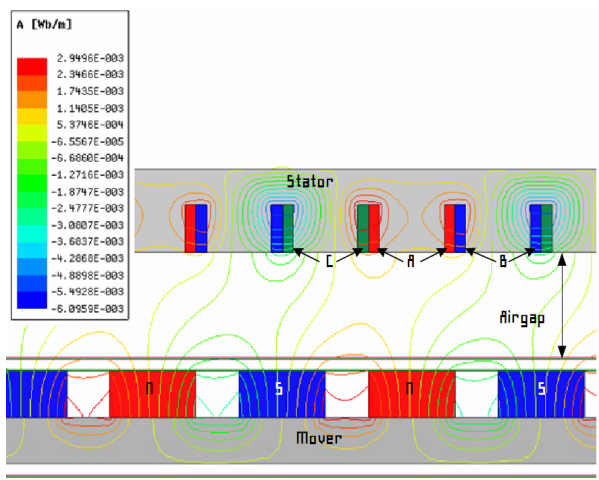

(a) The Magnetic vector potential

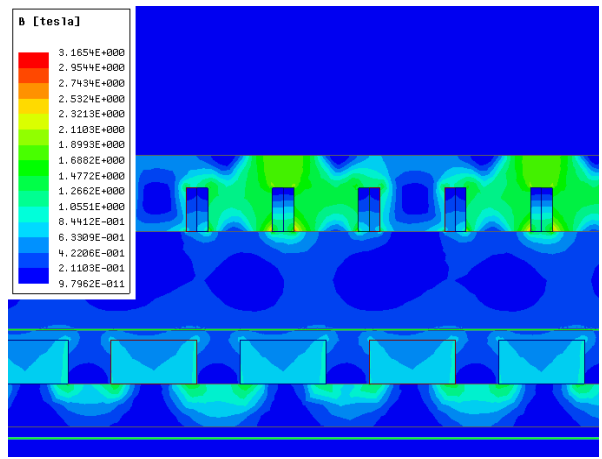

(b) The magnetic flux density

Fig. 2. FEM Analysis of propulsion vehicle

$$
\left[\begin{array}{l}
\Delta \dot{x}_{1}(t) \\
\Delta \dot{x}_{2}(t)
\end{array}\right]=A\left[\begin{array}{l}
\Delta x_{1}(t) \\
\Delta x_{2}(t)
\end{array}\right]+B_{\omega} \Delta F_{d}(t)+B_{u} \Delta u(t)
$$

where

$$
A=\left[\begin{array}{cc}
0 & 1 \\
\frac{\mu_{0} B^{2} A}{2 M} \frac{i_{0}^{2}}{z_{0}^{3}} & 0
\end{array}\right], B_{u}=\left[\begin{array}{c}
0 \\
-\frac{\mu_{0} N^{2} A}{2 M} \frac{i_{0}}{z_{0}^{2}}
\end{array}\right], B_{u}=\left[\begin{array}{c}
0 \\
\frac{1}{M}
\end{array}\right]
$$

It was found that the controllability of the system can be managed using the controllability matrix coefficient expressed in (5), unless the operating point of the input current is zero.

$$
C:=\left[\begin{array}{ll}
B & A B
\end{array}\right]=\left[\begin{array}{cc}
0 & -\frac{\mu_{0} N^{2} A}{2 M} \frac{i_{0}}{z_{0}^{2}} \\
-\frac{\mu_{0} N^{2} A}{2 M} \frac{i_{0}}{z_{0}^{2}} & 0
\end{array}\right]
$$

\section{FEM Analysis of Disturbance Forces of the LSM}

The disturbance force in the levitation direction occurs 


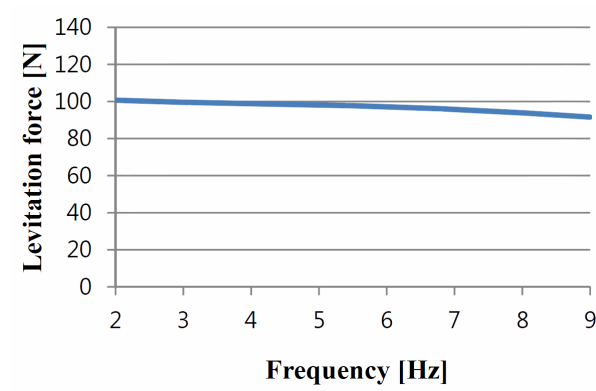

(a) Levitation Force by Frequency

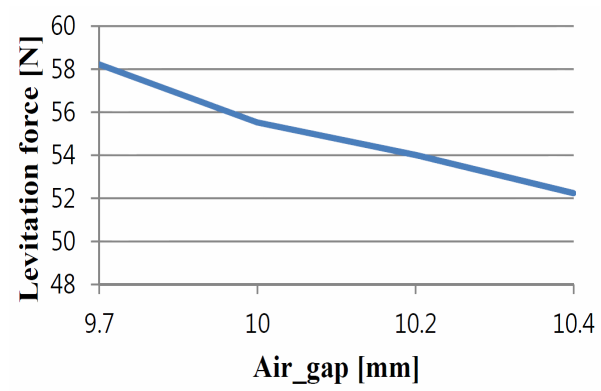

(b) Levitation Force by Air-Gap

Fig. 3. The spectrum of the disturbance force

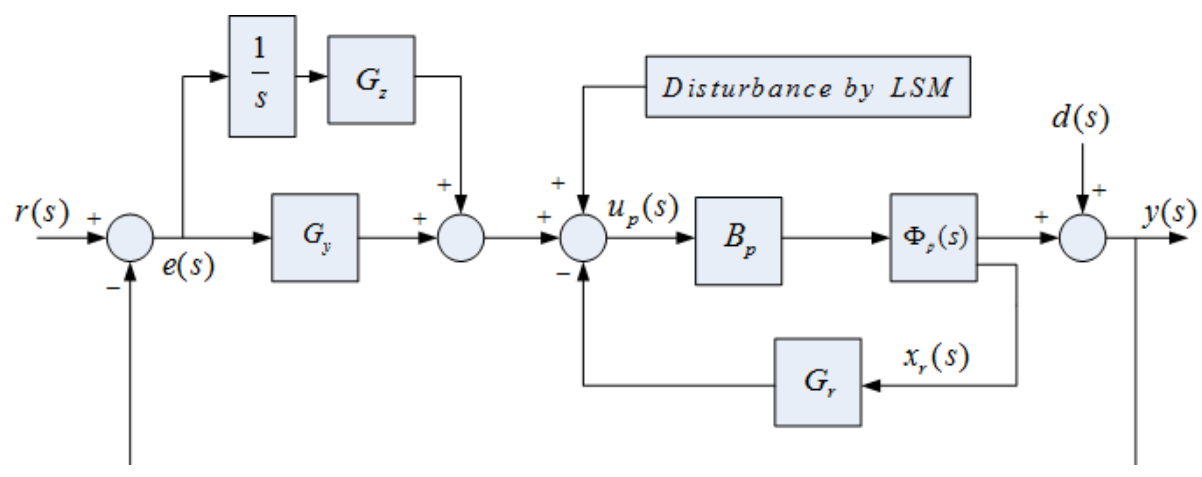

Fig 4. LQ-servo system considering the lsm normal force as disturbance

via the cogging torque of the propulsion LSM. Fig. 2 shows a two-dimensional analysis model of LSM where the total moving distance of the mover is set at $95 \mathrm{~mm}$ and the moving speed of the bogie is at a slow $500 \mathrm{~mm} / \mathrm{s}$. The material of the stator winding is iron, the number of turns per pole in the stator is 12 , and the stator current is applied at $4.5 \mathrm{~A}$.

The spectrum of the disturbance force dominantly from LSM is analyzed by FEM shown in Fig. 3. Fig. 3(a) shows the characteristics of the levitation force of LSM in a lowfrequency domain. In Fig. 3(b), the analyzed disturbance force varies with respect to the gap position.

\section{LQ-Servo Controller Design}

LQR (Linear Quadratic Regulator) is one of the popular optimal control methods, and guarantees the robustness and stability. A designated system that augments the output variable of the LQR system as a part of the state variable for a servo system design in order to develop tracking performance is known as the LQ servo system. It includes an integral element for the error signal because the LQ servo generates a steady-state error for the unit step reference [5]. As shown on the block diagram in Fig. 4, this system includes the disturbance generated by a normal LSM force.

Represented state variables of the LQ servo system, which includes the output variable and the state variable, are presented in Eq. (6).

$$
\begin{aligned}
& x(t)=\left[\begin{array}{lll}
z_{p}(t) & y_{p}(t) & x_{r}(t)
\end{array}\right]^{T}, \\
& \dot{x}(t)=A x(t)+B u(t) \\
& y(t)=C x(t)
\end{aligned}
$$

where $z_{p}(t)$ is the integral of the error, $y_{p}(t)$ is the plant output and $x_{r}(t)$ is the plant state variables. The state space matrices $\mathrm{A}, \mathrm{B}$ and $\mathrm{C}$ are as follows.

$$
A=\left[\begin{array}{cc}
0 & C_{p} \\
0 & A_{p}
\end{array}\right], \quad B=\left[\begin{array}{c}
0 \\
B_{p}
\end{array}\right], C=\left[\begin{array}{lll}
0 & I & 0
\end{array}\right],
$$

where $A_{p}$ is the state matrix, $B_{p}$ is the input matrix and $C_{p}$ is the output matrix of the plant.

The performance index $J$ and optimal control input $u(t)$ are presented in Eq. (8).

$$
\begin{aligned}
& J=\int_{0}^{\infty}\left[x^{T}(t) N^{T} B N x(t)+\rho u^{T}(t) u(t)\right] d t \\
& u(t)=-G x(t)=-G_{z} z_{p}(t)-G_{y} y_{p}(t)+G_{r} x_{p}(t)
\end{aligned}
$$

where $G=\left[\begin{array}{lll}G_{z} & G_{y} & G_{r}\end{array}\right]$ is LQR gain and $N=\left[N_{L} N_{H}\right]$ is the design matrix. The optimal control gain is determined in order to minimize the performance index with the 
weighting factor $N$.

A loop transfer function matrix $E(s)$ from error $e(t)$ to output $y(t)$, which is used to determine the frequency domain performance and stability-robustness of the LQ servo with an integral control element, and a feedback transfer function $G_{L Q}(s)$ are as follows:

$$
\begin{aligned}
& E(s)=C_{p}\left(s I-A_{p}-B_{p} G_{r} D_{p}\right)^{-1}\left[B G_{y}+\frac{1}{s} G_{z}\right] \\
& G_{L Q}(s)=G(s I-A)^{-1} B
\end{aligned}
$$

Generally, the tracking performance and the disturbance have energy in the low-frequency domain, and sensor noise has energy in the high-frequency domain [9]. Therefore, the control design should be conducted in the frequency domain for each performance. The design parameters $Q=N^{T} N$ and $R=\rho I$ can be calculated from an equivalent formula in the frequency domain. In order to determine the design parameter $N$, the loop transfer function of the controlled systems is presented as Eq. (10) with respect to the design factor in the frequency domain [10]

$$
\sigma_{i}\left[I+G_{L Q}(j \omega)\right]=\sqrt{1+\frac{1}{\rho} \sigma_{i}^{2}\left[N(j \omega I-A)^{-1} B\right]},
$$

where $N(s I-A)^{-1} B$ can be expressed as follows:

$$
\begin{aligned}
N(s I-A)^{-1} B & =N\left\{\left[\begin{array}{cc}
s I & 0 \\
0 & s I
\end{array}\right]-\left[\begin{array}{cc}
0 & C_{p} \\
0 & A_{p}
\end{array}\right]\right\}^{-1} B \\
& =\left[\begin{array}{ll}
N_{L} & N_{H}
\end{array}\right]\left[\begin{array}{cc}
\frac{1}{S} I & \frac{1}{S} C_{p}\left(s I-A_{p}\right)^{-1} \\
0 & \left(s I-A_{p}\right)^{-1}
\end{array}\right]\left[\begin{array}{c}
0 \\
B_{p}
\end{array}\right] \\
& =\frac{1}{s} N_{L} C_{p}\left(s I-A_{p}\right)^{-1} B_{p}+N_{H}\left(s I-A_{p}\right)^{-1} B_{p}
\end{aligned}
$$

$N(s I-A)^{-1} B$ in the low-frequency domain and highfrequency domain is asymptotically derived as follows:

$$
\left\{\begin{array}{l}
s \rightarrow 0, N(s I-A)^{-1} B \rightarrow \frac{1}{s} N_{L} C_{p}\left(s I-A_{p}\right)^{-1} B_{p} \\
s \rightarrow \infty, N(s I-A)^{-1} B \rightarrow \frac{1}{S} N_{H} B_{p}
\end{array}\right.
$$

By determining the control factor as Eq. (13) for the low and high frequency, respectively. the frequency-domain characteristics of the LQ-servo controlled system is obtained as Eq. (14)

$$
\left\{\begin{array}{l}
N_{L}=\left(C_{p}\left(-A_{p}\right)^{-1} B_{p}\right)^{-1} \\
N_{H}=\left(B_{p}^{T} B_{p}\right)^{-1} B_{p}^{T}
\end{array}\right.
$$

$$
\left\{\begin{aligned}
\omega \rightarrow 0, \sigma_{i}\left[G_{L Q}(j \omega)\right] & \rightarrow \frac{1}{\omega \sqrt{\rho}} \\
\omega \rightarrow \infty, \sigma_{i}\left[G_{L Q}(j \omega)\right] & \rightarrow \frac{1}{\omega \sqrt{\rho}}
\end{aligned}\right.
$$

The proposed LQ-servo control method can attenuate the effect of the disturbance force in LSM propulsion system since the frequency loop shape of the controlled system is specified by Eq. (14) [10].

\section{Simulation and Experimental Results}

To verify the proposed LQ servo controlled system, it

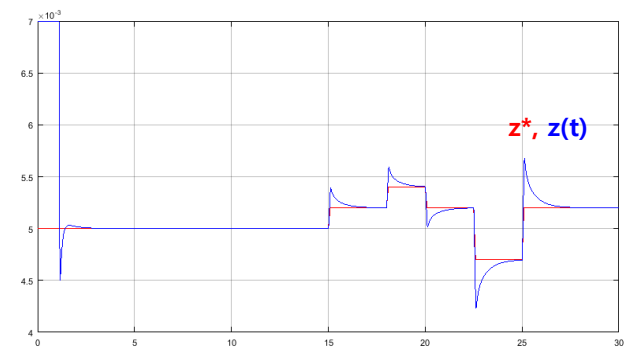

(a) PID : $z^{*} z(\mathrm{t}) /$ No disturbance

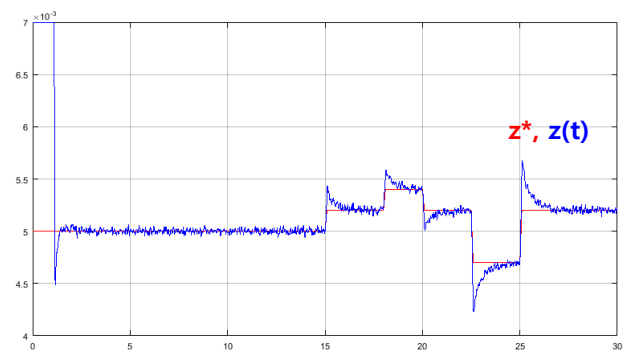

(b) PID : $z^{*}{ }_{-} \mathrm{z}(\mathrm{t}) /$ disturbance by LSM

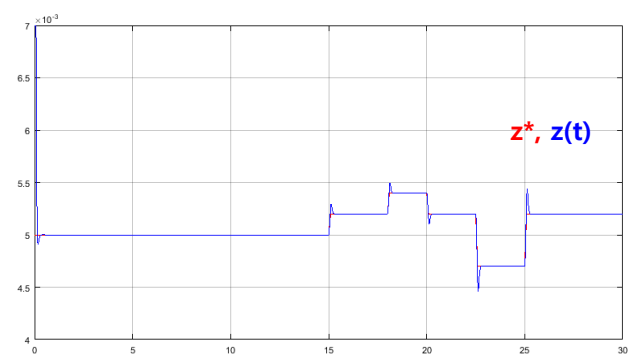

(c) LQ Servo : $z^{*} z(t) /$ disturbance NO

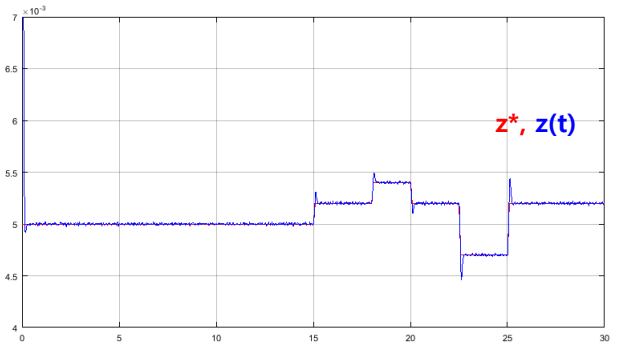

(d) LQ Servo : $z^{*} z(t) /$ disturbance by LSM

Fig. 5. The simulation results by controller and disturbance 
was compared with PID control via simulations and experiments.

\subsection{Simulation results}

In order to verify the performance of the proposed LQ servo controller, a simulation is performed using MATLAB/ Simulink. The system parameters are presented in Table 1, and Fig. 5 show the simulation results, which included the change in gap position.

As shown in Fig. 5, if there is a change in the gap, the LQ servo control has a much faster response and smaller overshoot than the PID control. In addition, if the disturbance of the normal force of LSM is taken into

Table 1. System parameters

\begin{tabular}{c|c|c}
\hline Parameter & Value & Unit \\
\hline $\mathrm{N}$ & 730 & turns \\
\hline $\mathrm{A}$ & 0.005 & $\mathrm{~m}^{2}$ \\
\hline $\mathrm{g}$ & 9.806 & $\mathrm{~m} / \mathrm{s}^{2}$ \\
\hline $\mathrm{M}$ & 17.5 & $\mathrm{~kg}$ \\
\hline$\mu_{0}$ & $4 \cdot \pi \cdot 10^{-7}$ & $\mathrm{H} / \mathrm{m}$ \\
\hline
\end{tabular}

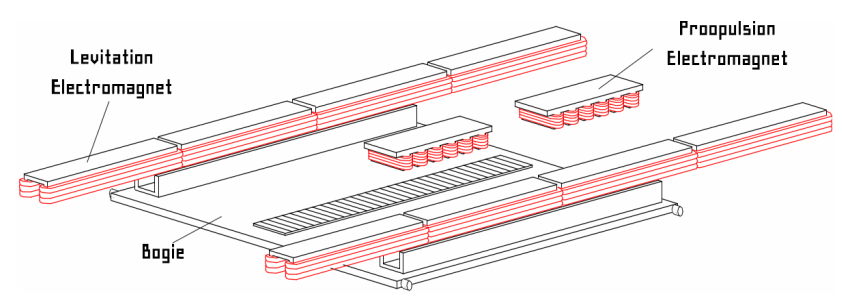

Fig. 6. Schematic of the magnetic levitation distribution transport system

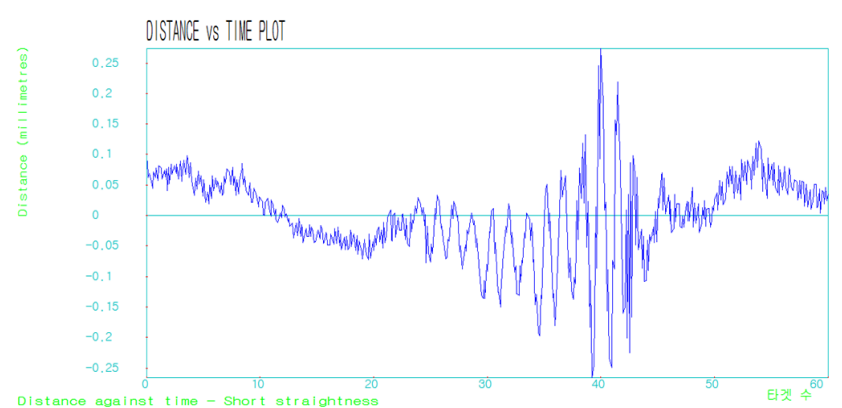

(a) PID: $z(t) /$ disturbance by LSM

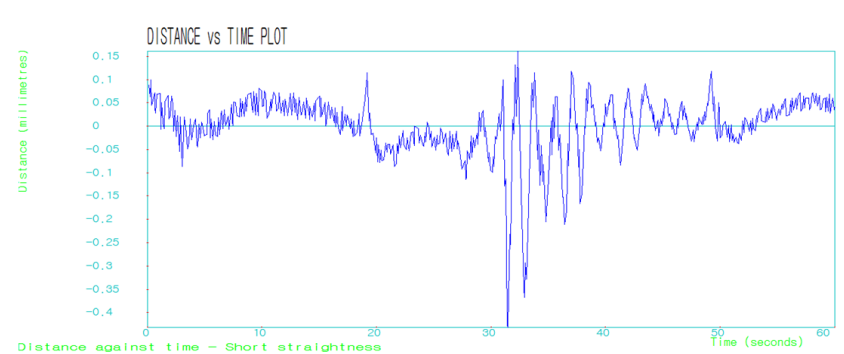

(b) LQ Servo: $z(t) /$ disturbance by LSM

Fig. 7. Experiment results account, the influence of the generated output is less effective. Therefore, the LQ servo control has a more stable performance compared to the PID Control.

\subsection{Experimental result}

As measuring equipment, a laser interferometer (Renishaw / XL-80) was used and the air-gap variation in the $\mathrm{z}$-axis direction was set as the object of measurement. The operating area is $350-1100 \mathrm{~mm}$ and is operated in forward and reverse. The speed was set at $10 \mathrm{~mm} / \mathrm{s}$ and the acceleration at $20 \mathrm{~mm} / \mathrm{s}^{2}$. The target was to make air-gap variation come within $-0.5 \mathrm{~mm}-0.5 \mathrm{~mm}$.

Fig. 7 shows the gap position of the bogie. Change of the gap for the PID Control System is observed from $-0.27 \mathrm{~mm}$ to $0.27 \mathrm{~mm}$, whereas change of the gap for the LQ Servo Control is observed from $-0.40 \mathrm{~mm}$ to $0.15 \mathrm{~mm}$.

\section{Conclusion}

In this paper, we developed an LQ-servo optimal control for the maglev system in order to avoid the frequency domain barrier, for good command following and noise rejection, by considering the disturbance force expected by FEM analysis of LSM propulsion system.

A mathematical model of the designated maglev system was developed to verify the simulation and a controller was implemented, which is resistant against disturbances. Controls using by the well-known PID System and LQ Servo System were performed and we compared the control performances against the disturbance. The simulation and experimental results indicate that the proposed LQ-Servo controlled system is more robust against disturbances than the conventional PID control.

\section{Acknowledgements}

This work was supported by the National Research Foundation of Korea(NRF) grant funded by the Korea government(Ministry of Science, ICT \& Future Planning) (No. 2016R1A2A1A05005392 and 2016R1A2B4009675).

\section{References}

[1] M. Morishita and T. Azukizawa, "A new maglev system for magnetically levitated carrier system," Veh. Technol. IEEE Trans., vol. 38, no. 4, 1989, pp. 230-236.

[2] H. W. Lee, K. C. Kim and J. Lee, "Review of Maglev Train Technologies," IEEE Trans. Magn., vol. 42, no. 7, Jul. 2006, pp. 1917-1925.

[3] C. H. Kim, Robust Control for In-Track Type Magnetic Levitation Systems, Ph.D Thesis, Hanyang 
University, 2015.

[4] J. H. Yang, LQ-PID Controller Design Method of the Frequency Domain Considering the Overshoot for the Second-order System, Ph.D Thesis, Hanyang University, 2010.

[5] C. H. Kim, H. J. Park, J. Lee, H. W. Lee, and K. D. Lee, "Multi-rate optimal controller design for electromagnetic suspension systems via linear matrix inequality optimization," Journal of Applied Physics, vol. 117, no. 17, May 2015, 17 B506.

[6] P. K. Sinha, Electromagnetic Suspension Dynamics \& Control, Peter Peregrinus Ltd., 1987.

[7] D. Trumper, "Linearizing control of magnetic suspension systems," IEEE Trans. Control Syst. Technol., vol. 5, no. 4, pp. 427-438, 1997.

[8] K. J. Kim, H. S. Han, C. H. Kim, and S. J. Yang, "Dynamic analysis of a maglev conveyor using an em-pm hybrid magnet," J. Electr. Eng. Technol., vol. 8, no. 6, Nov. 2013, pp. 1571-1578.

[9] H. Kwakernaak and R. Sivan, Linear Optimal Control System, $1^{\text {st }}$ ed. Wiley-Interscience, 1972.

[10] M. Athans, Multivarible Control System, Athan's Control Lecture Note, MIT.

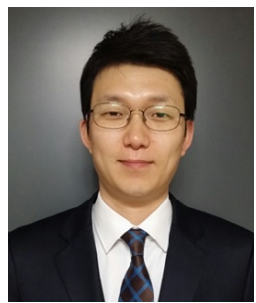

Chang-Hyun Kim $\mathrm{He}$ received his M.S and Ph.D in Electrical Engineering from Hanyang University, Korea, in 2006, and 2015, respectively. He was a senior researcher at Korea Marine Equipment Research Institute from 2015 to 2016 . He is currently an assistant professor at VISION College of Jeonju, Korea. His current research interests include the robust control, MPC, optimal digital control, and its application to electric power grid, network congestion control, magnetic levitation systems, and so on.

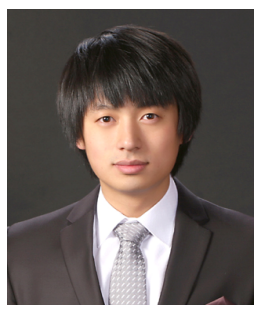

Hanwoong Ahn He received his B.S., M.S. and Ph.D. degrees in Electrical Engineering from Hanyang University, Seoul, Korea in 2010, 2012 and 2016, respectively. He is now working in Korea Aerospace Research Institute. His research interests include design, analysis, testing and control of motor/ generator; power conversion systems; and applications of motor drive such as electric vehicles, high-speed maglev train and renewable energy systems.

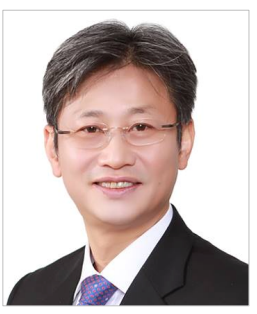

Ju Lee He received his M.S. degree from Hanyang University, Seoul, South Korea, in 1988, and his Ph.D. from Kyusyu University, Japan in 1997, both in Electrical Engineering, He joined Hanyang University in September, 1997 and is currently a Professor of the Division of Electrical and Biomedical Engineering. His main research interests include electric machinery and its drives, electromagnetic field analysis, new transformation systems such as hybrid electric vehicles (HEV), and high-speed electric trains and standardization. He is a member of the IEEE Industry Applications Society, Magnetics Society, and Power Electronics Society.

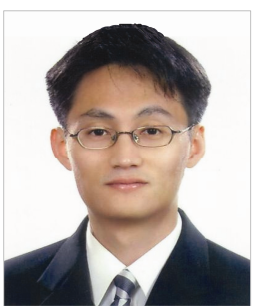

Hyungwoo Lee He received his M.S. degree from Hanyang University, Seoul, Korea, in 2000, and his Ph.D. from Texas A\&M University, College Station, TX, in 2003, both in Electrical Engineering. He joined Korea National University of Transportation as professor of the department of Railway Vehicle System Engineering in March 2013. His research interests include motor design, analysis of motor / generator; and applications of motor drive, such as Maglev trains, conventional railway propulsion systems, and modern renewable energy systems. 